\title{
Electroporation-Mediated Immunization of a Candidate DNA Vaccine Expressing Dengue Virus Serotype 4 prM-E Antigen Confers Long-Term Protection in Mice
}

\author{
Ziyang Sheng ${ }^{1} \cdot$ Hui Chen ${ }^{1} \cdot$ Kaihao Feng $^{1} \cdot \mathrm{Na} \mathrm{Gao}^{1} \cdot$ Ran Wang $^{1} \cdot$ Peigang Wang ${ }^{1} \cdot$ Dongying Fan $^{1} \cdot$ \\ Jing $\mathrm{An}^{1,2}$ (B)
}

Received: 17 October 2018/Accepted: 17 January 2019/Published online: 21 February 2019

(C) Wuhan Institute of Virology, CAS 2019

\begin{abstract}
Dengue fever, caused by dengue viruses (DENVs), is a widespread mosquito-borne zoonotic disease; however, there is no available anti-dengue vaccine for worldwide use. In the current study, a DNA vaccine candidate (pV-D4ME) expressing prM-E protein of DENV serotype 4 (DENV-4) was constructed, and its immunogenicity and protection were evaluated in immunocompetent BALB/c mice. The pV-D4ME candidate vaccine induced effective humoral and cellular immunity of mice against DENV-4 in vivo when administered both at $50 \mu \mathrm{g}$ and $5 \mu \mathrm{g}$ through electroporation. Two weeks after receiving three immunizations, both doses of $\mathrm{pV}$-D4ME DNA were shown to confer effective protection against lethal DENV-4 challenge. Notably, at 6 months after the three immunizations, $50 \mu \mathrm{g}$, but not $5 \mu \mathrm{g}$, of pV-D4ME could provide stable protection (100\% survival rate) against DENV-4 lethal challenge without any obvious clinical signs. These results suggest that immunization with $50 \mu \mathrm{g} \mathrm{pV}$-D4ME through electroporation could confer effective and long-term protection against DENV-4, offering a promising approach for development of a novel DNA vaccine against DENVs.
\end{abstract}

Keywords Dengue virus (DENV) · DNA vaccine $\cdot$ Long-term protection · Electroporation · Mouse

\section{Introduction}

Dengue virus (DENV), the pathogenic agent of dengue fever (DF), belongs to the family Flaviviridae, genus Flavivirus and is divided into four antigenically distinct, but closely related serotypes (DENV-1 to -4) (Guzman et al. 2010). Infection with any of the DENV serotypes in humans might cause DF with flu-like symptoms such as fever, rash, myalgia, and arthralgia, occasionally progressing into potentially lethal severe dengue (Rigau-Perez et al. 1998). During the past 5 decades, the burden of dengue has dramatically grown with a 30 -fold increase in the global incidence. The prevalence areas have also been

Jing An

anjing@ccmu.edu.cn

1 Department of Microbiology, School of Basic Medical Sciences, Capital Medical University, Beijing 100069, China

2 Center of Epilepsy, Beijing Institute for Brain Disorders, Beijing 100093, China expanding, and dengue is currently endemic in more than 100 countries (Ranjit and Kissoon 2011). It is estimated that approximately 390 million infections occur globally per year, 96 millions of which manifest clinical symptoms (Bhatt et al. 2013). Therefore, dengue has become a global public health problem.

However, there is no specific treatment or worldwide licensed vaccine available against DENV infection (Porter and Raviprakash 2015). Efforts toward the development of DF vaccines have resulted in several vaccine candidates that are currently in different clinical phases, such as TV003/TV005 developed by the National Institute of Allergy and Infectious Disease, and DENVax developed by Inviragen/Takeda (WHO 2016). Notably, the first licensed live recombinant tetravalent dengue vaccine (Dengvaxia ${ }^{\circledR}$, chimeric yellow fever-dengue virus tetravalent dengue vaccine, CYD-TDV), developed by Sanofi Pasteur, was registered for marketing in Mexico in December 2015 and was authorized for clinical use in individuals 9-45 years of age in nearly 20 endemic countries in Asia and Latin America, including Mexico, the Philippines, and Brazil 
(Pang et al. 2017; Fatima and Syed 2018). However, CYDTDV is not widely recommended owing to its partial efficacy and varied protection against certain types in some age groups, as well as the potential long-term risk of severe dengue (Hadinegoro et al. 2015; WHO 2017). More recently, significant safety concerns were raised in regards to Dengvaxia, and there is a potential risk that it could enhance infection even more efficiently than a wild-type dengue infection (Halstead 2016). Therefore, safer, economic, and more effective vaccines against dengue are urgently needed.

A DNA vaccine has several advantages, including costeffectiveness, ease of production, excellent stability, and high safety (Sardesai and Weiner 2011; Khan 2013). One of the main attractive features of a DNA vaccine is that it is capable of inducing strong cellular and humoral immune responses, which are both important for protection against DENVs in vivo (Wei et al. 2014; Suschak et al. 2015). However, their poor immunogenicity have limited the practical application of DNA vaccines to date (Kim et al. 2010). Several approaches have been proposed and investigated to overcome this issue, including use of an effective gene delivery system and immune adjuvants. Among of these, electroporation (EP)-mediated DNA immunization was demonstrated to enhance the uptake of exogenous antigens more effectively than conventional administration of the DNA vaccine, and could improve the immunogenicity and immune response, even in large animals, including humans (Hirao et al. 2008; Livingston et al. 2010; Sardesai and Weiner 2011). Chen et al. (2016) reported that EP could enhance the uptake of DNA plasmids by muscle cells due to the instant formation of micropores on the cellular membrane induced by the electric shock, which substantially promoted the gene delivery and efficiency of antigen expression in vivo. Moreover, EP-mediated immunization was shown to upregulate the expression of inflammatory genes and recruited immune cells such as dendritic cells, macrophages, and lymphocytes, which are involved in antigen processing and presentation (Babiuk et al. 2004).

Thus far, three DNA vaccines have been licensed for veterinary use, including two prophylactic vaccines against infectious hematopoietic necrosis virus in Atlantic salmon and West Nile virus in horses, and an immunotherapeutic vaccine for malignant melanoma in dogs (Liu 2011). Moreover, numerous EP studies in humans focusing on diverse clinical protocols, including cancer immune therapy (Yang et al. 2014) and prophylaxis of virus infections (Hutnick et al. 2011; Weiland et al. 2013; Stachyra et al. 2014), have confirmed the effectiveness of this platform. Thus, we hypothesized that administration of a DNA vaccine via EP could improve the immunogenicity of DNA vaccines and offer effective protection against DENV-4 infection as an ideal option for developing a novel dengue vaccine (Lambricht et al. 2016).

DENV is a single-stranded, positive-sense RNA virus and the genome encodes three structural proteins, capsid (C), premembrane/membrane (prM/M), and envelope (E) proteins, and seven non-structural (NS) proteins: NS1, NS2A, NS2B, NS3, NS4A, NS4B, and NS5. Amongst them, E protein has a direct correlation with the production of neutralizing antibody (NAb) in vivo, which makes it an optimal target antigen for vaccine development (Clements et al. 2010). The prM protein, the precursor of $M$ protein, functions as a chaperone protein that is essential for $\mathrm{E}$ protein conformation and secretion as well as viral maturation and exocytosis (Heinz et al. 1994; Ocazionez Jimenez and Lopes da Fonseca 2000). Therefore, prM-E is considered an ideal molecular target for developing DNA vaccines for Flavivirus, such as Japanese encephalitis virus (JEV), Zika virus (ZIKV) and DENVs (Chen et al. 2016; Sheng et al. 2016; Griffin et al. 2017).

Based on this background, in the present study, we constructed a DNA vaccine candidate by cloning fragments of the prM and E-encoding sequences of DENV-4 into pVAX1, a unique US Food and Drug Association-approved vector for DNA vaccine development (Huang et al. 2016; Dai et al. 2017). The constructed candidate vaccine, designated $\mathrm{pV}-\mathrm{D} 4 \mathrm{ME}$, was administered to $\mathrm{BALB} / \mathrm{C}$ mice via $\mathrm{EP}$ at two doses $(50 \mu \mathrm{g}$ and $5 \mu \mathrm{g})$, and the immunogenicity was evaluated by monitoring the immune response and consequence of DENV-4 challenge at 2 weeks and 6 months after the final of three immunizations. Our results might contribute to development of a novel DNA vaccine against DENVs.

\section{Materials and Methods}

\section{Cells, Virus, and Animals}

Vero cells were grown in minimum essential medium (Gibco, Australia) containing 5\% fetal bovine serum (FBS; PAN, Germany) at $37{ }^{\circ} \mathrm{C}$ with $5 \% \mathrm{CO}_{2}$. Aedes albopictus C6/36 cells were grown in RPMI 1640 medium (Gibco, Australia) supplemented with $10 \% \mathrm{FBS}$ at $28{ }^{\circ} \mathrm{C}$ with $5 \%$ $\mathrm{CO}_{2}$.

DENV-4 strain H241 (GenBank sequence accession number AY947539.1) was propagated in C6/36 cells and stored at $-70{ }^{\circ} \mathrm{C}$ until use. Virus titers in Vero cells were determined by a plaque assay with $1.2 \%$ methylcellulose overlay medium.

Six-week-old female inbred BALB/c mice were purchased from Vital River Laboratories (Beijing, China). All mice were maintained in specific-pathogen-free conditions. 


\section{Construction of the Recombinant Plasmid}

Dengue virus type 4 vector p4 (GenBank sequence accession number: AY648301.1) was used as the template to amplify fragments of the $p r M$ and $E$ genes containing signal peptides (369-2423 bp). A pair of specific primers with Kpn I/Xba I restriction sites (forward: 5'-CCGAC GGGTACCATGGTCATGCTGAACATCTT-3', reverse: 5'-GCGGGCTCTAGATTATGCTTGAACTGTGAAG-3'; restriction sites are underlined) was designed for PCR. The amplified fragments were digested with the Kpn I and $X b a$ I restriction enzymes (Invitrogen, USA) and subsequently subcloned into the multiple cloning sites of pVAX1 (Invitrogen, USA). The recombinant plasmid verified by enzyme digestion and DNA sequencing was designated $\mathrm{pV}$-D4ME (D4).

\section{Indirect Immunofluorescence Assay (IFA)}

IFA was used to confirm the expression of the recombinant plasmid in eukaryotic cells as described previously (Chen et al. 2012). In brief, pV-D4ME was transfected into a monolayer of Vero cells with Lipofectamine ${ }^{\mathrm{TM}} 2000$ (Lipo2000, Invitrogen, USA) in Opti-MEM ${ }^{\circledR}$ I Medium (Invitrogen, USA) and served as the antigen. Phosphatebuffered saline (PBS) containing 2\% bovine serum albumin was used to block non-specific binding. Mouse antiDENV4 polyclonal antibodies were produced in our lab and used as the primary antibody (1:100 dilution). FITCconjugated goat anti-mouse IgG (1:200, Immunotech, France) was applied as the secondary antibody. The results were assessed under a fluorescence microscope. The empty vector $\mathrm{pVAX} 1$ served as a negative control.

\section{DNA Immunization in Mice}

The mice were randomly divided into three experimental groups: $50 \mu \mathrm{g}$ (D4-50) or $5 \mu \mathrm{g}$ (D4-5) of $\mathrm{pV}-\mathrm{D} 4 \mathrm{ME}$ and $50 \mu \mathrm{g}$ of pVAX1 $(\mathrm{pV})$ as the control. For immunization, different doses $(50 \mu \mathrm{g}$ or $5 \mu \mathrm{g})$ of $\mathrm{pV}$-D4ME in $50 \mu \mathrm{L}$ sterile saline were inoculated into the quadriceps muscle of the hind limb of the mice through EP in a syringe with a 25 -gauge needle. Immediately, two silver electrodes $6 \mathrm{~mm}$ apart were inserted at the injection site and six electricpulse stimulations were applied at $36 \mathrm{~V}$ for $10 \mathrm{~ms}$ each using a Teresa Gene Delivery Device (Shanghai Teresa Healthcare Sci-Tech, China). The mice were immunized a total of three times at 2-week intervals. Mice inoculated with $50 \mu \mathrm{g}$ pVAX1 $(\mathrm{pV})$ via EP served as negative controls. Immunogenicity and protection were respectively assessed in each group of immunized mice.
Enzyme-Linked Immunosorbent Assay (ELISA)

The sera of immunized mice were collected by tail bleeding the day before immunization at different time points, and were analyzed for the presence of anti-DENV-4 antibodies with ELISA; sera of non-immune mice served as the negative control. Live DENV-4 virus was inactivated at $56{ }^{\circ} \mathrm{C}$ for $30 \mathrm{~min}$, and $10^{4}$ plaque-forming units (PFU) of the inactivated virus were coated per well in a 96-well plate as the antigen. Serial diluted serum samples were then added to each well and the plate was incubated at $4{ }^{\circ} \mathrm{C}$ overnight. After washing with PBS, secondary antibodies labeled with streptavidin-horseradish peroxidase (HRP) were added to each well. After washing and color development, the absorbance was read at $492 \mathrm{~nm}$ in a Multiskan Spectrum 1500 microplate reader (Thermo, USA). The end-point titers of the antibodies were determined as the reciprocal of the highest dilution, giving an optical density twice that of the non-immune serum. The geometric mean value of end-point titers (GMT) of antibodies in each group was calculated.

\section{Plaque Reduction Neutralization Test (PRNT)}

To determine the titers of NAb in the sera of immunized mice after three immunizations, sera were heated at $56{ }^{\circ} \mathrm{C}$ for $30 \mathrm{~min}$ to inactivate complements and diluted twofold starting at $1: 10$. Subsequently, $500 \mu \mathrm{L}$ of the serial dilutions were mixed with an equal volume of the DENV-4 suspension containing $1000 \mathrm{PFU}$ and incubated for $1 \mathrm{~h}$ at $37^{\circ} \mathrm{C}$. The mixtures were then added to each well of monolayer Vero cells in a 24-well plate, incubated, washed, and overlaid with complete medium containing $1.2 \%$ methylcellulose. After 7 days of further incubation, the plates were stained with crystal violet and the plaques were counted. The NAb titers were calculated as the reciprocal of the maximum dilution of serum that yielded a $50 \%$ plaque reduction (PRNT50) in comparison with that of the virus controls.

\section{Immunohistochemical (IHC) Staining}

To detect the expression of pV-D4ME in situ, the murine quadriceps muscles around the injection site were harvested 2 weeks after the final immunization, and prepared as paraffin-embedded sections for IHC. In brief, after dewaxing, blocking endogenous peroxidase activity, and retrieving antigens, sections were incubated with mouse anti-DENV4 polyclonal antibody (1:100) followed by incubation with HRP-conjugated goat anti-mouse IgG. The sections were then color-developed with 3,3'-diaminobenzidine and counterstained with hematoxylin. After 
mounting with resin, the sections were examined under a light microscope (Olympus BX51, Japan).

\section{Enzyme-Linked Immunospot Assay (ELISPOT)}

Splenocytes obtained aseptically from the mice were subjected to a cytokine assay with ELISPOT sets (BD Biosciences, USA) following the manufacturer's instructions. Specifically, the levels of interferon (IFN)- $\gamma$, interleukin (IL)-2, IL-4, and IL-10 were determined to evaluate the immune responses in vivo. In brief, splenocytes were placed on 96-well filtration plates pre-coated with capture Abs at $10^{6}$ cells/well (Millipore, USA) and then stimulated with full DENV-4 inactivated particles at $10 \mu \mathrm{g} / \mathrm{well}$ at $37{ }^{\circ} \mathrm{C}$ for $60 \mathrm{~h}$. Splenocytes stimulated with concanavalin A served as a positive control, and RPMI 1640 medium alone served as a negative control. After incubation with biotinylated detection Abs followed by streptavidin-HRP, spots at the sites of cytokine secretion were developed by adding 3-amino-9-ethylcarbazole substrate and were counted automatically by an ELISPOT reader system (CTL, USA).

\section{Active Protection against DENV-4 Challenge}

Two weeks or six months after the final immunization, the mice were challenged intracranially with a lethal dose of DENV-4 ( $10^{4}$ PFU per mouse). The body weight, complication development, and mortality were monitored for 21 days. The clinical signs were scored according to the following ranking criteria: 0 , healthy; 1 , ruffled hair or hunchbacked appearance; 2, asthenia, wasting, or bradykinesia; 3 , forelimb or hindlimb weakness; 4, paralysis or moribundity; and 5, death.

\section{Statistical Analysis}

Statistical analysis was conducted with SPSS 17.0 software. Levels of NAb were calculated as the GMT after reciprocal transformation. Survival curves were estimated by the Kaplan-Meier method with the log-rank test for significance assessment. Weight changes and clinical sign scores were analyzed by repeated-measures analysis of variance (ANOVA). Other data were compared using oneway ANOVA. $P<0.05\left(^{*}\right)$ and $P<0.01 \quad(* *)$ were regarded as a significant difference and highly significant difference, respectively.

\section{Results}

\section{Expression of pV-D4ME In Vitro and In Vivo}

To determine the expression of $\mathrm{pV}-\mathrm{D} 4 \mathrm{ME}$ in eukaryotic cells, plasmids were transfected into Vero cells. Cells infected with DENV-4 served as positive control. The $\mathrm{pV}$ D4ME-transfected Vero cells showed positive fluorescence, while no specific fluorescence was observed in the cells transfected with the control pVAX1 vector (Fig. 1A), indicating effective expression of the prM and E proteins of DENV-4.

Furthermore, the expression of pV-D4ME in muscular tissues of immunized mice was determined by IHC. Strong specific immunoreactivity was observed in the cytoplasm of mouse muscle cells 2 weeks after the last immunization in the D4-50 group (Fig. 1B), while only weakly positive immunoreactivity was detected in the D4-5 group. No immunoreactivity developed in the $\mathrm{pV}$ group, indicating that pV-D4ME could be effectively expressed in situ by electro-mediated DNA uptake.

\section{Humoral Immune Responses in pV-D4ME- Immunized Mice}

Two weeks after three immunizations, the humoral immune response, including specific $\operatorname{IgG}$ and $\mathrm{NAb}$, induced by $\mathrm{pV}$-D4ME was determined. As shown in Fig. 2, both $50 \mu \mathrm{g}$ and $5 \mu \mathrm{g} \mathrm{pV}-\mathrm{D} 4 \mathrm{ME}$ elicited the production of specific IgG antibody against DENV-4 with a GMT of 1:2540 and 1:566, respectively, representing a significant difference $(P<0.05)$. However, there was no detectable specific $\mathrm{IgG}$ production in the control $\mathrm{pV}$ group. Consistently, specific NAb with a GMT of 1:45.7 was observed in the D4-50 group, which was a significantly higher titer than that observed in the D4-5 group $(P<0.01)$. Only half of the mice $(3 / 6)$ produced a borderline level of NAb (1:10), and the rest failed to develop detectable NAb. No specific NAb titer was detected in the $\mathrm{pV}$ group. This result indicated that the DNA-elicited antibody response displayed a dose-dependent pattern, and $5 \mu \mathrm{g} \mathrm{pV}$-D4ME was insufficient to induce the production of $\mathrm{NAb}$ at a protective level.

\section{Cytokine Production in pV-D4ME-Immunized Mice}

To determine cellular immune response, levels of splenocyte-derived cytokines were determined by ELISPOT at 2 weeks after three immunizations. Significant increases in the levels of IFN- $\gamma$, IL-2, IL-4, and IL-10 were detected in the D4-50 group compared to those of the $\mathrm{pV}$ group 
Fig. 1 Expression of pV-D4ME in vitro and in vivo. (A) DENV4 antigens expression in plasmid-transfected Vero cells. The pV-D4ME (D4)- or pVtransfected Vero cells were cultured separately for $48 \mathrm{~h}$ and then the expression was detected by IFA. Cells infected with DENV-4 served as a positive control. Bar $=100 \mu \mathrm{m}$. (B) DENV-4 antigens expression in the quadriceps femoris muscle of BALB/c mice immunized with $50 \mu \mathrm{g}$ (D4-50) or $5 \mu \mathrm{g}$ (D4-5) of pV-D4ME. Samples were harvested at 2 weeks after the last of the three immunizations and the antigen was detected by IHC. Bar $=50 \mu \mathrm{m}$.
A
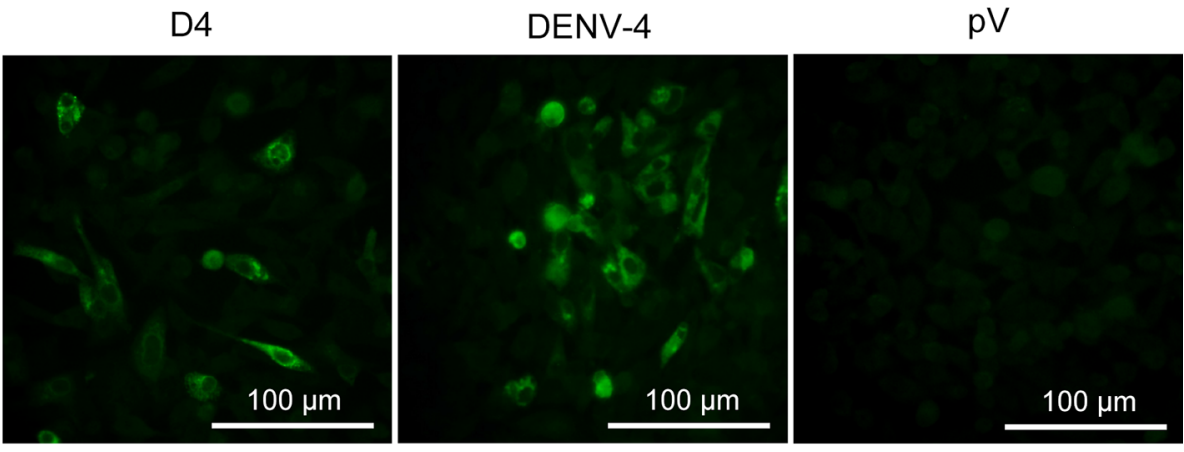

B

D4-50

D4-5

$\mathrm{pV}$
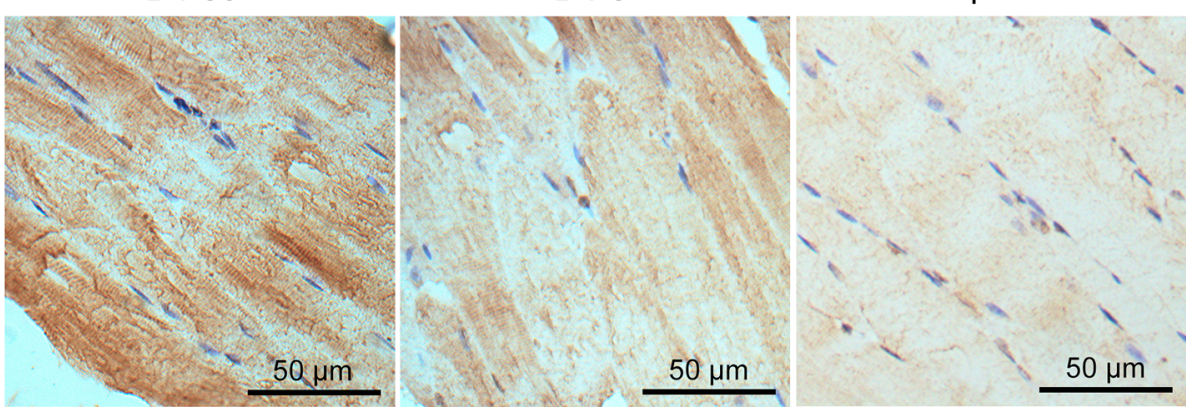

A

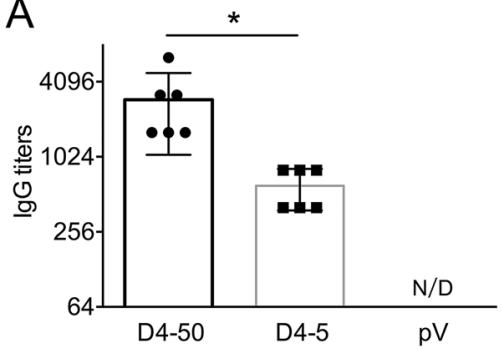

B

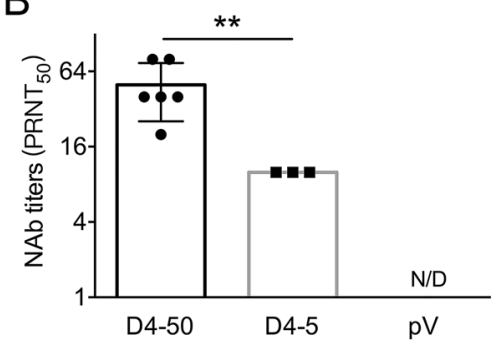

Fig. 2 DENV-4-specific antibody responses in sera of immunized $\mathrm{BALB} / \mathrm{c}$ mice 2 weeks after three immunizations. Endpoint titers of (A) IgG and (B) NAb determined by ELISA and PRNT, respectively. The reciprocals of titers are represented as the mean $\pm \mathrm{SD} . \mathrm{n}=6$. N/D, not detected. Titers were log-transformed and analyzed by twoway ANOVA. $* P<0.05 ; * * P<0.01$.

( $P<0.01$, Fig. 3). However, there was relatively less of an increase in these cytokines in the D4-5 group, implying a limited immune response (Fig. 3).

\section{pV-D4ME Provided Protection Against Lethal DENV-4 Challenge in Mice}

Immunized mice were challenged with $10^{4} \mathrm{PFU}$ of DENV4 individually at 2 weeks after three immunizations to determine the efficiency of vaccine. As shown in Fig. 4, mice in the control group, clinical signs was observed and body weight loss was noted at 7 days post-infection (dpi). The symptoms gradually progressed, and all mice died within 12-18 dpi. In contrast, the survival rates of mice immunized with $50 \mu \mathrm{g}$ and $5 \mu \mathrm{g} \mathrm{pV-D4ME} \mathrm{were}$ $100 \%(6 / 6)$ and $83 \%(5 / 6)$, respectively. No obvious clinical manifestations were observed in the D4-50 group, and there were no differences in survival rates or clinical signs between the high- and low-dose groups.

Similarly, following DENV-4 challenge at 6 months after the last immunization, mice in the control group showed the same pattern of disease progression as observed for the control mice challenged after 2 weeks (Fig. 5). In addition, all of the mice in the D4-50 group survived without symptoms, whereas only $60 \%$ (3/5) of mice in the D4-5 group survived and one of the survivors had severe paralysis of the hindquarters. This result indicated that $50 \mu \mathrm{g}$ of $\mathrm{pV}$-D4ME is necessary for inducing long-term protection against DENV-4.

To understand the mechanisms underlying the long-term protection, sera were collected from the mice before and after challenge, and the specific IgG and NAb titers were determined. At 6 months after vaccinations before the challenge, the specific IgG and NAb titers were maintained 
Fig. 3 Levels of splenocytesecreted cytokines in immunized $\mathrm{BALB} / \mathrm{c}$ mice at 2 weeks after three immunizations detected by ELISPOT. (A) IFN- $\gamma$, (B) IL-2, (C) IL-4, and (D) IL-10 levels. The numbers of cytokinepositive cells are shown as the mean counts of spot-forming unit $(\mathrm{SFU}) \pm \mathrm{SD}$ per $1 \times 10^{6}$ splenocytes. $\mathrm{n}=6$. Results were analyzed by the $t$ test. $* P<0.05 ; * * P<0.01$.
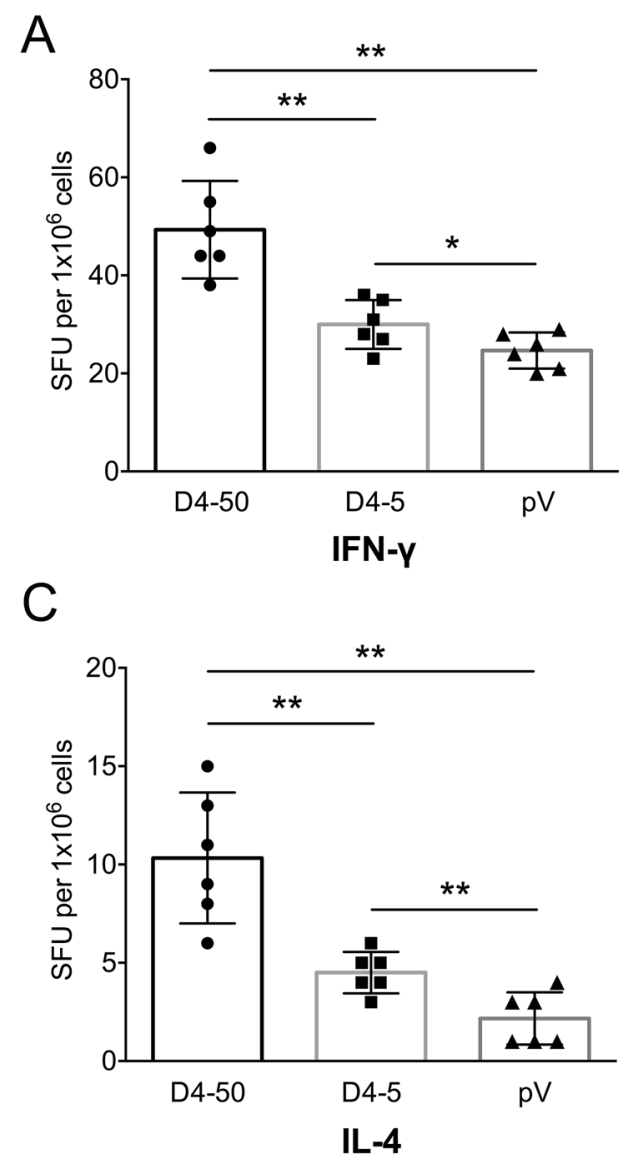

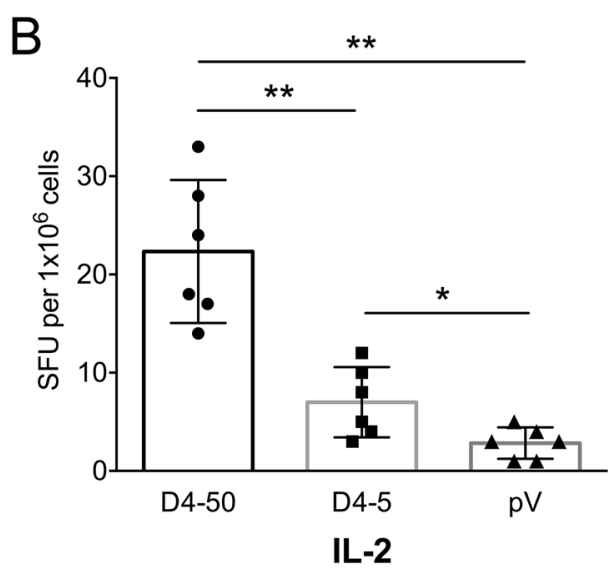

D

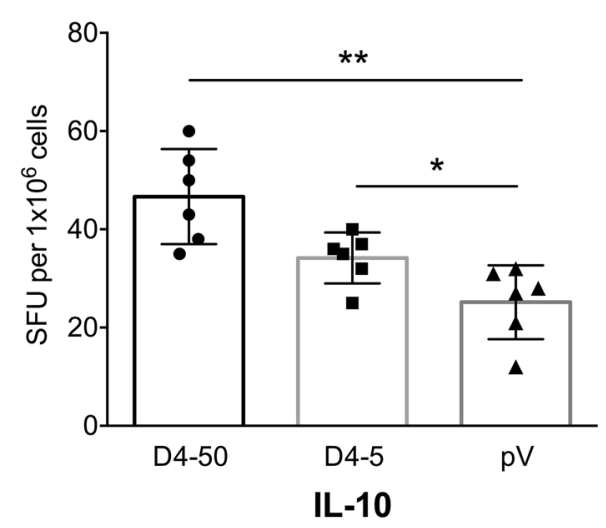

at the same level as those measured at 2 weeks after immunization. The IgG titers were 1:3676 and 1:459 in the D4-50 and D4-5 groups, respectively. The NAb titer increased to 1:53 in the D4-50 group, whereas there was detectable NAb in only one mouse (1/5) of the D4-5 group. Interestingly, after DENV-4 challenge, the IgG titers significantly elevated to $1: 7352$ and 1:635 in the D4-50 and D4-5 group, respectively. The NAb titer increased to 1:139 in the D4-50 group and to 1:16 in the surviving mice of the D4-5 group. This obviously enhanced antibody response after challenge indicated the excellent immune memory in pV-D4ME-immunized mice (Fig. 6).

\section{Discussion}

In the decades following the discovery of DENV, various approaches were proposed for developing vaccines against dengue, including an inactivated virus vaccine, live attenuated virus vaccine, chimeric virus vaccine, subunit vaccines, vectored vaccines, and DNA vaccines (Yauch and Shresta 2014). Unfortunately, there is still no widely used vaccine against dengue, although CYD-TDV has been registered and licensed in several endemic countries. Here, we demonstrate that the newly constructed candidate DNA vaccine $\mathrm{pV}$-D4ME could induce specific humoral and cellular immune responses in mice after three immunizations at $50 \mu \mathrm{g}$, and the immunized mice were completely protected from DENV-4 challenge even after 6 months. Collectively, these findings indicate $\mathrm{pV}-\mathrm{D} 4 \mathrm{ME}$ as a promising vaccine candidate against dengue serotype 4 .

Cellular and humoral immune responses are considered to be equally important for prevention against dengue infection as a safe and efficient vaccine (Zellweger et al. 2013). In our study, IFN- $\gamma$, IL-2, IL-4, and IL-10 were all markedly boosted in the D4-50 group, accompanied by a $100 \%$ survival rate. The Th1 response is mediated by Th1 helper cells with production of IFN- $\gamma$ and IL-2, which are associated with the cellular immune response, while the Th2 response is closely related with the production of IL-4 and IL-10, which boosts the humoral response. Three doses of the vaccine candidate $\mathrm{pV}$-D4ME at $50 \mu \mathrm{g}$ could elicit balanced cellular and humoral responses as well as complete protection. However, when the dosage of the immunogen was reduced to $5 \mu \mathrm{g}$, immunized mice showed apparent clinical symptoms, limited levels of NAb and the cytokines, along with a reduced survival rate (83\%) against DENV-4 challenge, implying weakened immune responses and protection. Therefore, both the humoral and cellular immune response showed an antigen dose-dependent 

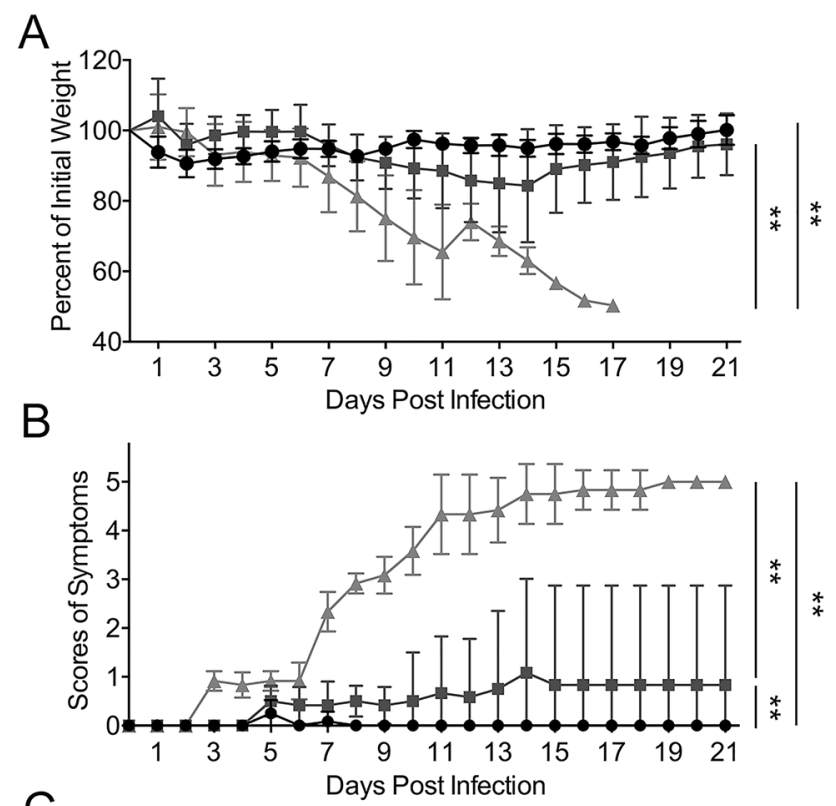

C

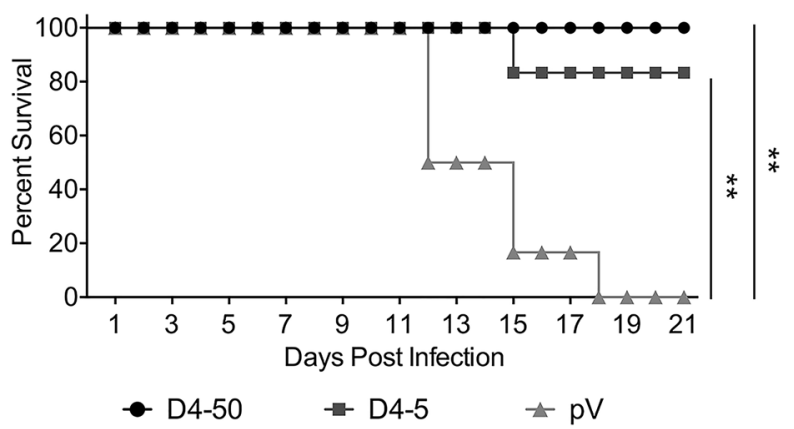

Fig. 4 Protective immunity elicited by pV-D4ME against DENV-4 challenge in $\mathrm{BALB} / \mathrm{c}$ mice at 2 weeks after three immunizations. Mice were challenged with $10^{4}$ PFU DENV-4 and monitored daily for 21 days. (A) Percentage of body weight change compared to day 0 . (B) Scores of illness symptoms. (C) Survival rates. Data are presented as mean $\pm \mathrm{SD}$ in $(\mathbf{A})$ and $(\mathbf{B}) . \mathrm{n}=6$. The percentage of body weight and the scores of illness symptoms were analyzed by two-way ANOVA. The survival rates were analyzed by the Kaplan-Meier method. $* * P<0.01$.

pattern, and $50 \mu \mathrm{g} \mathrm{pV}$-D4ME is necessary for inducing effective cellular and humoral immune responses and protection under this experimental condition.

The long-term effectiveness of DNA vaccines is a general concern that requires assessment for all candidates. We found effective protection of $50 \mu \mathrm{g}$ pV-D4ME via EP up to 6 months after the last immunization with no symptoms detected in any of the mice, while all of the mice in the control group died. Accordingly, high levels of IgG and $\mathrm{NAb}$ were observed before the challenge, which were dramatically elevated after DENV-4 challenge in the D450 group, but not in the D4-5 group. Indeed, the low dosage $(5 \mu \mathrm{g})$ of pV-D4ME only conferred limited protection with a reduced survival rate compared to the high-dose group, and severe hindlimb paralysis observed. Moreover, our

A

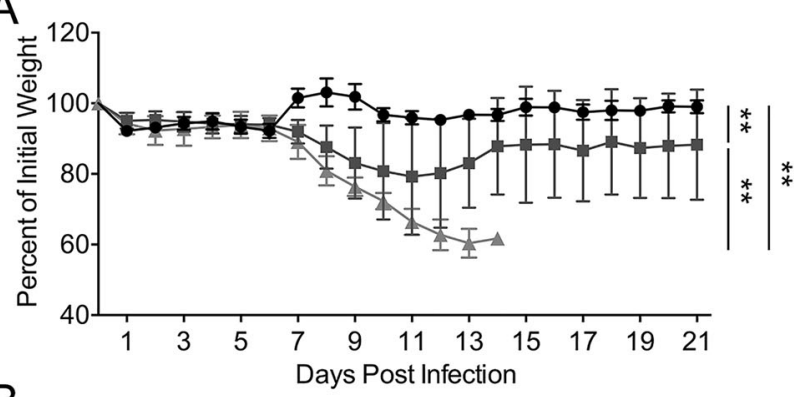

B

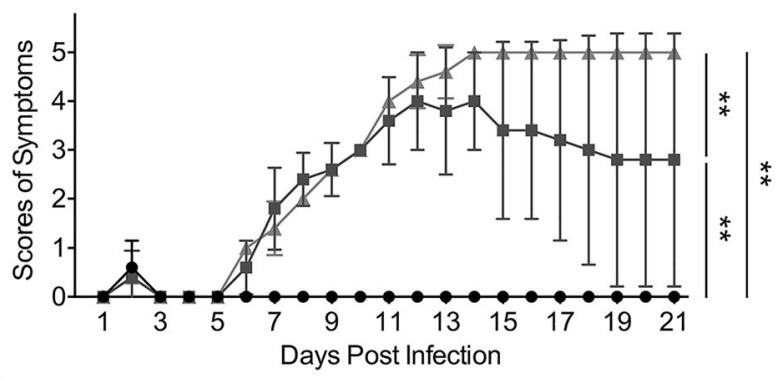

C

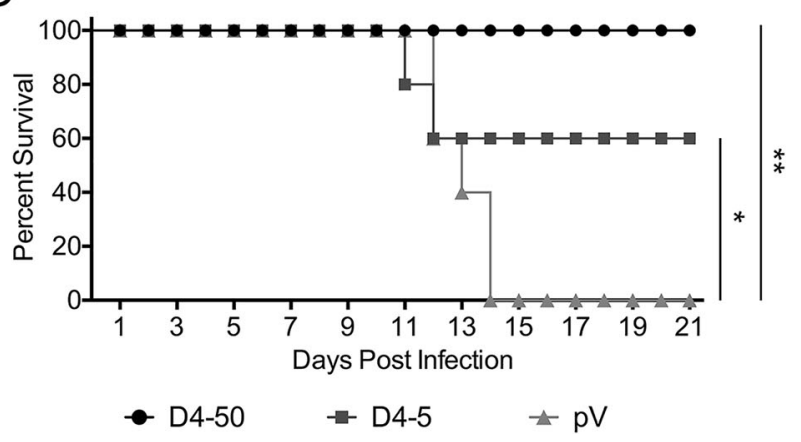

Fig. 5 Long-term protection elicited by pV-D4ME against DENV-4 infection in $\mathrm{BALB} / \mathrm{c}$ mice at 6 months after three immunizations. Mice were challenged with $10^{4}$ PFU DENV-4 at 6 months after three immunizations and monitored daily for 21 days. (A) Percentage of body weight change compared to day 0. (B) Scores of illness symptoms. (C) Survival rates. Data are presented as mean \pm SD in (A) and (B). $n=5$. The percentage of body weight and the scores of illness symptoms were analyzed by two-way ANOVA. The survival rates were analyzed by the Kaplan-Meier method. $* * P<0.01$.

findings confirm that in vivo $\mathrm{EP}$ is a useful method for delivery that dramatically affects the magnitude and maintenance of the immune responses and could achieve effective protection. In our previous research on pCAGJME, a DNA vaccine candidate for JE, EP has been proved to be effective in increasing antigen expression and boosting immune response as compared with intramuscular injection, which might lengthen the maintaining of specific immunity (Sheng et al. 2016). Hence, in this study, in vivo EP is considered to be one of the critical factors contributing to the long-term protection with a sufficient amount of the immunogen.

Balanced protection against all four serotype DENVs is a key factor in development of tetravalent DNA vaccines 


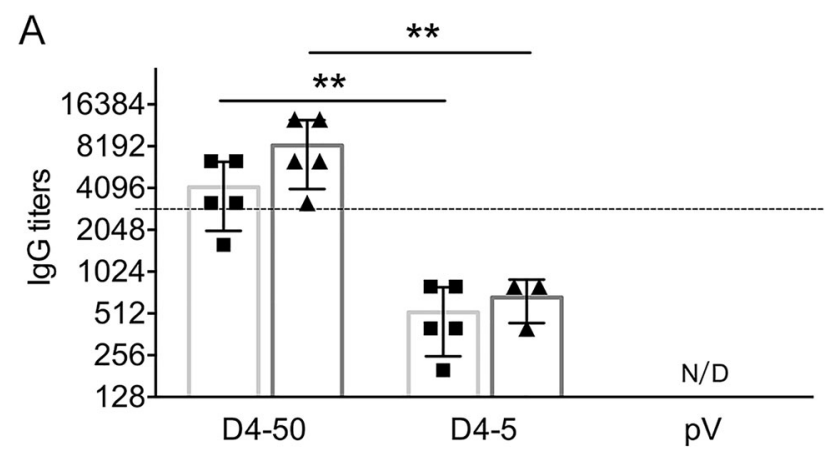

B

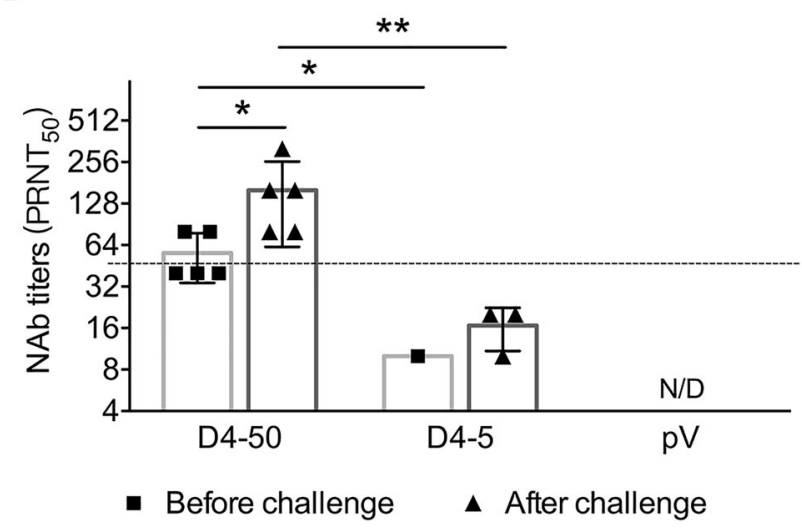

Fig. 6 DENV-4-specific antibody responses in sera of immunized BALB/c mice at 6 months after three immunizations. Endpoint titers of (A) $\operatorname{IgG}$ and (B) NAb determined by ELISA and PRNT, respectively, at 6 months after three immunizations. To identify the memory immunization, antibody titers were also determined in surviving mice at 21 days after DENV-4 challenge. The reciprocals of titers are presented as mean \pm SD. $n=5$. N/D, not detected. The dotted line refers to the titers of IgG or NAb of immunized mice at 2 weeks after three immunizations. Log-transformed titers were analyzed by two-way ANOVA. $* P<0.05 ; * * P<0.01$.

against dengue. Thus, one of the limitations of the present study is the lack of evaluation of the cross-protection of $\mathrm{pV}$-D4ME to the other three serotypes of dengue. We previously demonstrated that immunization with a bivalent vaccine consisting of $\mathrm{pV}-\mathrm{D} 1 \mathrm{ME}$ and $\mathrm{pV}-\mathrm{D} 2 \mathrm{ME}$ via $\mathrm{EP}$ generated balanced and effective humoral responses along with protection against both DENV1 and DENV2 in mice (Zheng et al. 2017). Recently, we used the same strategy to immunize mice with tetravalent vaccines consisting of $\mathrm{pV}$ D1ME, pV-D2ME, pV-D3ME, and pV-D4ME. Preliminary results revealed that the immunization could provide a balanced humoral immune response to DENV1-4, indicating possible balanced protection against the four serotypes. However, confirmation and detailed experiments are ongoing.

In summary, we have demonstrated that pV-D4ME administered via EP in vivo is capable of triggering sufficient and balanced cellular and humoral immune responses and long-term protection against DENV-4 infection in mice, offering a promising new option for developing DNA vaccines against dengue.

Acknowledgements This work was supported by the National Natural Science Foundation of China (81772172, 81471957, 81671971, U1602223).

Author Contributions ZS, HC, KF, NG, DF performed the experiments. RW and PW analyzed the data. JA organized the collaboration and prepared the manuscript. All authors read and approved the final manuscript.

\section{Compliance with Ethical Standards}

Conflict of interest The authors declare that they have no conflict of interest.

Animal and Human Rights Statement The whole study was approved by the Institutional Animal Care and Use Committee of Capital Medical University, China (Approval Number: AEEI-2015-066). All institutional and national guidelines for the care and use of laboratory animals were followed.

\section{References}

Babiuk S, Baca-Estrada ME, Foldvari M, Middleton DM, Rabussay D, Widera G, Babiuk LA (2004) Increased gene expression and inflammatory cell infiltration caused by electroporation are both important for improving the efficacy of DNA vaccines. J Biotechnol 110:1-10

Bhatt S, Gething PW, Brady OJ, Messina JP, Farlow AW, Moyes CL, Drake JM, Brownstein JS, Hoen AG, Sankoh O, Myers MF, George DB, Jaenisch T, Wint GR, Simmons CP, Scott TW, Farrar JJ, Hay SI (2013) The global distribution and burden of dengue. Nature 496:504-507

Chen H, Gao N, Fan D, Wu J, Zhu J, Li J, Wang J, Chen Y, An J (2012) Suppressive effects on the immune response and protective immunity to a JEV DNA vaccine by co-administration of a GM-CSF-expressing plasmid in mice. PLoS ONE 7:e34602

Chen H, Zheng X, Wang R, Gao N, Sheng Z, Fan D, Feng K, Liao X, An J (2016) Immunization with electroporation enhances the protective effect of a DNA vaccine candidate expressing prME antigen against dengue virus serotype 2 infection. Clin Immunol 171:41-49

Clements DE, Coller BA, Lieberman MM, Ogata S, Wang G, Harada KE, Putnak JR, Ivy JM, McDonell M, Bignami GS, Peters ID, Leung J, Weeks-Levy C, Nakano ET, Humphreys T (2010) Development of a recombinant tetravalent dengue virus vaccine: immunogenicity and efficacy studies in mice and monkeys. Vaccine 28:2705-2715

Dai Z, Huang J, Lei X, Yan Y, Lu P, Zhang H, Lin W, Chen W, Ma J, Xie Q (2017) Efficacy of an autophagy-targeted DNA vaccine against avian leukosis virus subgroup. J. Vaccine 35:808-813

Fatima K, Syed NI (2018) Dengvaxia controversy: impact on vaccine hesitancy. J Glob Health 8:010312

Griffin BD, Muthumani K, Warner BM, Majer A, Hagan M, Audet J, Stein DR, Ranadheera C, Racine T, De La Vega MA, Piret J, Kucas S, Tran KN, Frost KL, De Graff C, Soule G, Scharikow L, Scott J, McTavish G, Smid V, Park YK, Maslow JN, Sardesai NY, Kim JJ, Yao XJ, Bello A, Lindsay R, Boivin G, Booth SA, Kobasa D, Embury-Hyatt C, Safronetz D, Weiner DB, Kobinger 
GP (2017) DNA vaccination protects mice against Zika virusinduced damage to the testes. Nat Commun 8:15743

Guzman MG, Halstead SB, Artsob H, Buchy P, Farrar J, Gubler DJ, Hunsperger E, Kroeger A, Margolis HS, Martinez E, Nathan MB, Pelegrino JL, Simmons C, Yoksan S, Peeling RW (2010) Dengue: a continuing global threat. Nat Rev Microbiol 8:S7-16

Hadinegoro SR, Arredondo-Garcia JL, Capeding MR, Deseda C, Chotpitayasunondh T, Dietze R, Muhammad Ismail HI, Reynales H, Limkittikul K, Rivera-Medina DM, Tran HN, Bouckenooghe A, Chansinghakul D, Cortes M, Fanouillere K, Forrat R, Frago C, Gailhardou S, Jackson N, Noriega F, Plennevaux E, Wartel TA, Zambrano B, Saville M, Group C-TDVW (2015) Efficacy and long-term safety of a dengue vaccine in regions of endemic disease. N Engl J Med 373:1195-1206

Halstead SB (2016) Dengue vaccine efficacy: not a zero sum game. J Infect Dis 214:2014

Heinz FX, Stiasny K, Puschner-Auer G, Holzmann H, Allison SL, Mandl CW, Kunz C (1994) Structural changes and functional control of the tick-borne encephalitis virus glycoprotein $\mathrm{E}$ by the heterodimeric association with protein prM. Virology 198:109-117

Hirao LA, Wu L, Khan AS, Satishchandran A, Draghia-Akli R, Weiner DB (2008) Intradermal/subcutaneous immunization by electroporation improves plasmid vaccine delivery and potency in pigs and rhesus macaques. Vaccine 26:440-448

Huang T, Zhao K, Zhang Z, Tang C, Zhang X, Yue B (2016) DNA vaccination based on pyolysin co-immunized with IL-1beta enhances host antibacterial immunity against Trueperella pyogenes infection. Vaccine 34:3469-3477

Hutnick NA, Myles DJ, Bian CB, Muthumani K, Weiner DB (2011) Selected approaches for increasing HIV DNA vaccine immunogenicity in vivo. Curr Opin Virol 1:233-240

Khan KH (2013) DNA vaccines: roles against diseases. Germs 3:26-35

Kim D, Hung CF, Wu TC, Park YM (2010) DNA vaccine with alphagalactosylceramide at prime phase enhances anti-tumor immunity after boosting with antigen-expressing dendritic cells. Vaccine 28:7297-7305

Lambricht L, Lopes A, Kos S, Sersa G, Preat V, Vandermeulen G (2016) Clinical potential of electroporation for gene therapy and DNA vaccine delivery. Expert Opin Drug Deliv 13:295-310

Liu MA (2011) DNA vaccines: an historical perspective and view to the future. Immunol Rev 239:62-84

Livingston BD, Little SF, Luxembourg A, Ellefsen B, Hannaman D (2010) Comparative performance of a licensed anthrax vaccine versus electroporation based delivery of a PA encoding DNA vaccine in rhesus macaques. Vaccine 28:1056-1061

Ocazionez Jimenez R, Lopes da Fonseca BA (2000) Recombinant plasmid expressing a truncated dengue-2 virus $\mathrm{E}$ protein without co-expression of prM protein induces partial protection in mice. Vaccine 19:648-654

Pang T, Mak TK, Gubler DJ (2017) Prevention and control of denguethe light at the end of the tunnel. Lancet Infect Dis 17:e79-e87

Porter KR, Raviprakash K (2015) Nucleic acid (DNA) immunization as a platform for dengue vaccine development. Vaccine 33:7135-7140

Ranjit S, Kissoon N (2011) Dengue hemorrhagic fever and shock syndromes. Pediatr Crit Care Med 12:90-100

Rigau-Perez JG, Clark GG, Gubler DJ, Reiter P, Sanders EJ, Vorndam AV (1998) Dengue and dengue haemorrhagic fever. Lancet 352:971-977

Sardesai NY, Weiner DB (2011) Electroporation delivery of DNA vaccines: prospects for success. Curr Opin Immunol 23:421-429

Sheng Z, Gao N, Cui X, Fan D, Chen H, Wu N, Wei J, An J (2016) Electroporation enhances protective immune response of a DNA vaccine against Japanese encephalitis in mice and pigs. Vaccine 34:5751-5757

Stachyra A, Gora-Sochacka A, Sirko A (2014) DNA vaccines against influenza. Acta Biochim Pol 61:515-522

Suschak JJ, Wang S, Fitzgerald KA, Lu S (2015) Identification of Aim2 as a sensor for DNA vaccines. J Immunol 194:630-636

Wei J, Chen H, An J (2014) Recent progress in dengue vaccine development. Virol Sin 29:353-363

Weiland O, Ahlen G, Diepolder H, Jung MC, Levander S, Fons M, Mathiesen I, Sardesai NY, Vahlne A, Frelin L, Sallberg M (2013) Therapeutic DNA vaccination using in vivo electroporation followed by standard of care therapy in patients with genotype 1 chronic hepatitis C. Mol Ther 21:1796-1805

WHO (2016) Questions and answers on dengue vaccines. https:// www.who.int/immunization/research/development/dengue_q_ and_a/en/. Last updated 20 April 2018

WHO (2017) Dengue vaccine: WHO position paper, July 2016recommendations. Vaccine 35:1200-1201

Yang B, Jeang J, Yang A, Wu TC, Hung CF (2014) DNA vaccine for cancer immunotherapy. Hum Vaccines Immunother 10:3153-3164

Yauch LE, Shresta S (2014) Dengue virus vaccine development. Adv Virus Res 88:315-372

Zellweger RM, Miller R, Eddy WE, White LJ, Johnston RE, Shresta S (2013) Role of humoral versus cellular responses induced by a protective dengue vaccine candidate. PLoS Pathog 9:e1003723

Zheng X, Chen H, Wang R, Fan D, Feng K, Gao N, An J (2017) Effective protection induced by a monovalent DNA vaccine against dengue virus (DV) serotype 1 and a bivalent DNA vaccine against DV1 and DV2 in mice. Front Cell Infect Microbiol 7:175 